\title{
ON SOME FAMILIES OF ANALYTIC FUNCTIONS ON RIEMANN SURFACES
}

\author{
SHINJI YAMASHITA
}

Dedicated to Professor K. Noshiro on the occasion of his 60th birthday

1. Throughout this paper all functions are single-valued. Let $R$ be a Riemann surface. We shall denote by $\varphi^{\wedge}$ the least harmonic majorant of a function $\varphi$ defined in $R$ if it has the meaning. We define the families $H_{p}(R)$ (for $p>0)$ and $S(R)(=D(R)$ in [17]) of analytic functions in $R$ by the following:

$f$ is in $H_{p}(R)$ if and only if the subharmonic function $|f|^{p}$ has a harmonic majorant in $R$;

$f$ is in $S(R)$ if and only if the subharmonic function $\log ^{+}(|f| / \mu)$ has a harmonic majorant in $R$ for some positive constant $\mu$ (and consequently for all $\mu>0)$ and $\left(\log ^{+}(|f| / \mu)\right)^{\wedge}\left(z_{0}\right) \rightarrow$ as $\mu \rightarrow+\infty$, where $z_{0}$ is a fixed point in $R$ ([17]).

We shall call $H_{p}=H_{p}(R)$ (resp. $S=S(R)$ ) the Hardy class (resp. the Smirnov class) in $R$.

A harmonic function $u$ in $R$ is said to be quasi-bounded ([13]) if it can be represented as: $u=u_{1}-u_{2}$, where $u_{j}(j=1,2)$ is the limiting function of a monotone non-decreasing sequence of non-negative and bounded harmonic functions in $R$.

A closed polar set $E$ in a Riemann surface $R$ is a closed set in $R$ such that for every open parameter disc $V$ in $R$, there exists a superharmonic function $s_{V}>0$ defined in $V$ with the property that $s_{V}=+\infty$ at every point in $V \cap E$, or equivalently, $V \cap E$ is a set of capacity zero in $V$ ([1], [2]). It is known that $R-E$ is connected.

Tumarkin and Havinson [17] (resp. Parreau [13]) investigated the null set $E$ in a plane domain (resp. in a Riemann surface) $R$ for the class $S$ (resp. $H_{p}$ ) under the condition that $E$ is a compact set of logarithmic capacity zero (resp. a closed, not necessarily compact, polar set) and proved: if an analytic function $f$ defined in $R-E$ belongs to the class $S(R-E)$

Received March 20, 1967 
(resp. $H_{p}(R-E)$ ), then there exists an analytic function $\tilde{f}$ defined in $R$ belonging to the class $S(R)$ (resp. $H_{p}(R)$ ) such that the restriction of $\tilde{f}$ to $R-E$ coincides with $f$.

In this paper we shall show, using the notion of quasi-bounded harmonic functions, that in these theorems the well-known fact that the closed polar set $E$ is removable for bounded and harmonic functions ([1], [2]) is essential.

As for S-part we shall prove the following:

Theorem 1. Any analytic function $f$ in a Riemann surface $R$ belongs to the Smirnov class $S(R)$ if and only if the subharmonic function $\log ^{+}|f|$ has a quasibounded harmonic majorant in $R$.

Using a version of Gårding and Hörmander's theorem [7] as a lemma, we shall prove:

Theorem 2. Any analytic function $f$ in a Riemann surface $R$ belongs to the Hardy class $H_{p}(R)$ (for $p>0$ ) if and only if the subharmonic function $|f|^{p}$ has a quasi-bounded harmonic majorant in $R$.

Seeing the above characterizations for the two classes, we are tempted to say the following:

THEOREM 3. Let $\Psi(r)$ be a continuous extended real-valued function defined for $r \geq 0$ satisfying the condition that for any finite positive real number $c$, the set of $r$ such that the inequality $\Psi(r) \leq c$ holds is bounded (from above). Let $R$ be a Riemann surface, $E$ be a closed polar set lying in $R$ and $f$ be an anaylytic function defined in $R-E$ such that the composite function $\Psi(|f|)$ has a quasi-bounded harmonic majorant in $R-E$.

Then there exists an analytic function $\tilde{f}$ defined in $R$ such that the composite function $\Psi(|\tilde{f}|)$ has a quasi-bounded harmonic majorant in $R$ and the restriction of $\tilde{f}$ to $R-E$ coincides with the function $f$.

As corollaries we have an extension of Tumarkin-Havinson's theorem and a new proof of Parreau's.

At the end, we shall give an example for the classification theory of open Riemann surfaces, which admits a non-constant analytic Lindelöfian function [9] and no non-constant analytic function in the Smirnov class.

2. Let $R$ be a Riemann surface, $H P^{\prime}(R)$ be the family of all the har- 
monic functions $u$ in $R$ such that the subharmonic function $|u|$ has a harmonic majorant in $R$. It is well-known (see for example, [3]) that $H P^{\prime}(R)$ forms a vector lattice under the lattice operations:

$$
\begin{aligned}
& u \vee v=(\text { the least harmonic majorant of } \max (u, v)) ; \\
& u \wedge v=-(-u) \vee(-v)
\end{aligned}
$$

for $u, v$ in $H P^{\prime}(R)$. For $u$ in $H P^{\prime}(R)$ we define $M u$ as follows:

$$
M u=u \vee 0-u \wedge 0 .
$$

We know that $M u=u \vee(-u)$ and $M(M u)=M u$. A function $u$ in $H P^{\prime}(R)$ is, by definition, quasi-bounded if

$$
M u=\lim _{n \rightarrow+\infty}(M u) \wedge n
$$

or equivalently,

$$
\lim _{n \rightarrow+\infty}(M u-n) \vee 0=0,
$$

where $n$ are positive numbers which can be considered as elements in $H P^{\prime}(R)$ and the limit is taken in the sense of the lattice operation, namely, $(M u) \wedge n$ (resp. $(M u-n) \vee 0)$ tends to $M u$ (resp. 0) non-decreasingly (resp. nonincreasingly) in $R$. A function $u$ in $H P^{\prime}(R)$ is called singular if

$$
\lim _{n \rightarrow+\infty}(M u) \wedge n=0 .
$$

It is shown by Parreau [13] that any $u$ in $H P^{\prime}(R)$ can be decomposed uniquely as:

$$
u=u_{B}+u_{S},
$$

where $u_{B}$ is quasi-bounded and $u_{S}$ is singular. The operator $u \rightarrow u_{B}$ (resp. $\left.u \rightarrow u_{S}\right)$ from $H P^{\prime}(R)$ into itself is linear, positive, i.e., $u \geq 0$ implies $u_{B} \geq 0$ (resp. $u_{S} \geq 0$ ) and idempotent, i.e., $\left(u_{B}\right)_{B}=u_{B}$ (resp. $\left.\left(u_{S}\right)_{S}=u_{S}\right)$. Of course, $u$ is quasi-bounded (resp. singular) if and only if $u_{S}=0$ (resp. $\left.u_{B}=0\right)$.

In the remainder of this paper we shall assume that the Riemann surface $R$ is hyperbolic since the situation is obvious in the parabolic case.

A subharmonic function $v$ in $R$ having a harmonic majorant in $R$ can be decomposed uniquely as:

$$
v=v^{\wedge}-p
$$


where $v^{\wedge}$ is the least harmonic majorant of $v$ and $p \geq 0$ is a Green's potential in $R$ (F. Riesz's decomposition).

We shall say that a subharmonic function $v$ in $R$ is quasi-bounded if $v^{\text {^ }}$ in the above decomposition is in $H P^{\prime}(R)$ and quasi-bounded. A subharmonic function $v$ having a quasi-bounded harmonic majorant $u$ and a quasibounded harmonic minorant $w$ simultaneously is quasi-bounded for $0=w_{S} \leq\left(v^{\wedge}\right)_{S} \leq u_{S}=0$. Especially, a non-negative subharmonic function is quasi-bounded if and only if it has a quasi-bounded harmonic majorant.

Let $\left\{R_{n}\right\}_{n=1}^{\infty}$ be a normal exhaustion of $R$ in Pfluger's sense, $\partial R_{n}=\Gamma_{n}$ be the boundary of $R_{n}$ (consisting of a finite number of piecewise analytic closed Jordan curves), $z_{0}$ be a fixed point in $R_{1}$ and $\omega_{n, z_{0}}$ be the harmonic measure of $\Gamma_{n}$ with respect to the domain $R_{n}$ measured at the point $z_{0}$ (for $n=1,2, \ldots)$. Then obviously we have:

$$
v^{\wedge}\left(z_{0}\right)=\lim _{n \rightarrow+\infty} \int_{\Gamma_{n}} v(z) d \omega_{n, z_{0}}(z) .
$$

An extended real-valued function $f(z)$ defined for points $z$ in $R$ is said to be uniformly absolutely integrable with respect to the system $\left\{\left(\Gamma_{n}, \omega_{n, z_{0}}\right)\right\}_{n=1}^{\infty}$ (we shall say simply "U.A.I. for $z_{0}$ and $\left\{R_{n}\right\}$ ") if the followings are satisfied:

$$
\sup _{n} \int_{\Gamma_{n}}|f(z)| d \omega_{n, z_{0}}(z)<\infty
$$

and

(b) for any $\varepsilon>0$, there exists a $\delta>0$ such that

$$
\left|\int_{A_{n}} f(z) d \omega_{n, z_{0}}(z)\right|<\varepsilon
$$

uniformly for $n=1,2, \ldots$, if only $A_{n} \subset \Gamma_{n}$ and $\omega_{n, z_{0}}\left(A_{n}\right)<\delta$.

According to de la Vallée Poussin [18] and Doob [4], [6], a function $f(z)$ in $R$ is U.A.I. for $z_{0}$ and $\left\{R_{n}\right\}$ if and only if there exists a non-negative monotone non-decreasing convex function $\Phi(r)$ defined for $r \geq 0$ satisfying the conditions:

$$
\lim _{r \rightarrow+\infty} \Phi(r) / r=+\infty
$$

and

$$
\sup _{n} \int_{\Gamma_{n}} \Phi(|f(z)|) d \omega_{n, z_{0}}(z)<\infty
$$


We shall call this de la Vallée Poussin-Doob's lemma.

In particular, if a subharmonic function $v(z) \geq 0$ in $R$ is U.A.I. for $z_{0}$ and $\left\{R_{n}\right\}$, then the condition (ii) above can be read as:

(ii)' The subharmonic function $\Phi(v)$ has a harmonic majorant in $R$.

We state some lemmas which will be used later.

LEMma 1. Let $v$ be a quasi-bounded subharmonic function in a Riemann surface $R$. Then $v$ is U.A.I. for arbitrary point $z_{0}$ in $R$ and arbitrary exhaustion $\left\{R_{n}\right\}, z_{0}$ in $R_{1}$. Conversely assume that a subharmonic function $v$ in $R$ is U.A.I. for at least one point $z_{0}$ and at least one exhaustion $\left\{R_{n}\right\}, z_{0}$ in $R_{1}$. Then $v$ is a quasi-bounded subharmonic function in $R$.

Proof. We know that any harmonic function belongs to $H P^{\prime}(R)$ and is quasi-bounded if and only if it is U.A.I. for one point $z_{0}$ and for one exhaustion $\left\{R_{n}\right\}, z_{0}$ in $R_{1}$ (and consequently for all) (see [4]). It is easy to check that Green's potential $p \geq 0$ is always U.A.I. for $z_{0}$ and $\left\{R_{n}\right\}$ since

$$
\int_{\Gamma_{n}} p(z) d \omega_{n, z_{0}}(z) \rightarrow 0 \text { as } n \rightarrow+\infty .
$$

Using the above two facts, we have immediately the assertions.

Lemma 2. A subharmonic function $v$ is quasi-bounded if and only if there exists a non-negative monotone non-decreasing convex function $\Phi(r)$ defined for $r \geq 0$ satisfying the conditions (i) and (ii).

Proof. This is a consequence of de la Vallée Poussin-Doob's lemma and Lemma 1.

3. Here we remark the relations between some families of analytic functions defined in a Riemann surface $R$. We define the families $A B(R)$ and $A L(R)$ of analytic functions in $R$ by the following:

$f$ is in $A B(R)$ if and only if $|f|$ is bounded in $R$;

$f$ is in $A L(R)$ if and only if the subharmonic function $\log ^{+}|f|$ has a harmonic majorant in $R$.

Then the following inclusion relations:

$$
A B(R) \subset H_{p}(R) \subset S(R) \subset A L(R) \quad(\text { for } p>0)
$$

are proved by the inequalities: 


$$
\log ^{+}(|f| / \mu) \leq|f|^{p} /\left(p \cdot \mu^{p}\right)
$$

and

$$
\log ^{+}|f| \leq \log ^{+}(|f| / \mu)+\log ^{+} \mu
$$

REMARK. The functions $f$ in the class $A L(R)$ are Lindelöfian analytic functions in the sense of Heins [9] and in the special case where $R$ is the unit open disc, are analytic functions of bounded type in Nevanlinna's sense [12]. The Smirnov class $S(R)$ was first investigated by V.I. Smirnov [16].

Now we give

Proof of Theorem 1. Let $\mu \geq 1$. Then we obtain

$$
\log ^{+}(|f| / \mu)=\max \left(\log ^{+}|f|-\log \mu, 0\right) .
$$

Consequently we have

$$
\begin{aligned}
\left(\log ^{+}(|f| / \mu)\right)^{\wedge} & =\left(\max \left(\log ^{+}|f|-\log \mu, 0\right)\right)^{\wedge} \\
& =\left(\max \left(\left(\log ^{+}|f|\right)^{\wedge}-\log \mu, 0\right)\right)^{\wedge} \\
& =\left(\left(\log ^{+}|f|\right)^{\wedge}-n\right) \vee 0,
\end{aligned}
$$

where $n=\log \mu$ and $\varphi^{\wedge}$ is the least harmonic majorant of $\varphi$ (see $\S 1$ ). Hence the condition that

$$
\left(\log ^{+}(|f| / \mu)\right)^{\wedge}\left(z_{0}\right) \rightarrow 0 \text { as } \mu \rightarrow+\infty
$$

is equivalent to the condition that

$$
\lim _{n \rightarrow+\infty}\left(\left(\log ^{+}|f|\right)^{\wedge}-n\right) \vee 0=0
$$

by Harnack's theorem, or $\left(\log ^{+}|f|\right)^{\wedge}$, the least harmonic majorant of $\log ^{+}|f|$, is quasi-bounded. Q.E.D.

REMARK. It is easy to show that $\log ^{+}|f|$ has a quasi-bounded harmonic majorant in $R$ if and only if $\log |f|$ has a quasi-bounded harmonic majorant in $R$.

By Lemma 1 with $v=\log ^{+}|f|$ and by Theorem 1 we have

Corollary 1. (An extended form of Theorem 1 in [17]) Any analytic function $f$ is in the Smirnov class $S(R)$ if and only if the subharmonic function $\log ^{+}|f|$ is U.A.I. for arbitrary fixed point $z_{0}$ in $R$ and arbitrary exhaustion $\left\{R_{n}\right\}, z_{0}$ in $R_{1}$. 
Corollary 2. (An extended form of Theorem 2 in [17]) Any analytic function $f$ is in the Smirnov class $S(R)$ if and only if the subharmonic function $\log ^{+}|f|$ has a harmonic majorant which is U.A.I. for arbitrary fixed point $z_{0}$ in $R$ and arbitrary exhaustion $\left\{R_{n}\right\}, z_{0}$ in $R_{1}$.

The following corollary shows that Gehring's class $N^{*}$ in [8] is a special case of the Smirnov class $S(R)$ where $R$ is the unit open disc.

Corollary 3. Any analytic function $f$ is in the class $S(R)$ if and only if there exists a non-negative monotone non-decreasing convex function $\Phi(r)$ satisfying the condition (i) in $\S 2$ and the subharmonic function $\Phi\left(\log ^{+}|f|\right)$ has a harmonic majorant in $R$.

Proof. This is a consequence of Theorem 1, Lemma 2 and (ii)' in $\S 2$.

4. In this section we shall study the Hardy class $H_{p}(R)$.

Let $\Delta$ be Martin's boundary of a hyperbolic Riemann surface $R$ and $\Delta_{1}$ be the totality of minimal points on $\Delta$. Let $K(z, \zeta)$ be Martin's kernel with respect to the fixed reference point $z_{0}$ in $R$, namely, $K\left(z_{0}, \zeta\right)=1$ for any point $\zeta$ in $R \cup \Delta$. Then it is known that to any function $u$ in the family $H P^{\prime}(R)$, there corresponds a unique signed Baire measure $d \mu$ on $\Delta_{1}$ of total mass finite such that

$$
u(z)=\int_{\Delta_{1}} K(z, \zeta) d \mu(\zeta)
$$

Let $d \omega$ be the measure on $\Delta_{1}$ corresponding to the constant function 1 , that is,

$$
1=\int_{\Delta_{1}} K(z, \zeta) d \omega(\zeta)
$$

for any point $z$ in $R$. Any function $u$ in $H P^{\prime}(R)$ has the fine limit $\left.u^{*}(\zeta) 1\right)$ at $d \omega$-almost every point $\zeta$ in $\Delta_{1}$ and the quasi-bounded part $u_{B}$ of $u$ is given by

$$
u_{B}(z)=\int_{\Delta_{1}} K(z, \zeta) u^{*}(\zeta) d \omega(\zeta)
$$

On the contrary, the singular part $u_{s}$ of $u$ in $H P^{\prime}(R)$ is represented as

1) In this section we shall denote by $u^{*}$ the fine limit of any function $u$ if it has the meaning. 


$$
u_{S}(z)=\int_{\Delta_{1}} K(z, \zeta) d \mu_{S}(\zeta)
$$

where $d \mu_{S}$ is a singular measure on $\Delta_{1}$ with respect to $d \omega$ and $u_{S}$ has the fine limit zero at $d \omega$-almost every point in $\Delta_{1}$. In conclusion:

$$
d \mu(\zeta)=u^{*}(\zeta) d \omega(\zeta)+d \mu_{\varsigma}(\zeta)
$$

$u^{*}$ is integrable with respect to $d \omega$.

Let $v$ be a subharmonic function in $R$ and have a harmonic function in $H P^{\prime}(R)$ as a majorant. Then F. Riesz's decomposition of $v$ becomes:

$$
v=v^{\wedge}-p,
$$

where, in this case, $v^{\wedge}$ is in $H P^{\prime}(R)$. Green's potential $p$ has the fine limit zero at $d \omega$-almost every point in $\Delta_{1}$. Consequently we may write in this case

$$
v^{*}=\left(v^{\wedge}\right)^{*}=\left(\left(v^{\wedge}\right)_{B}\right)^{*} .
$$

As to the notion of the fine limit at Martin's compactification, see Naïm [11] and Doob [5].

Now we are ready to state a generalization of Gårding and Hörmander's theorem ([7]). ${ }^{2)}$

Lemma 3. Let $v$ be a subharmonic function defined in $R$. Let $\varphi(r)$ be a non-negative monotone non-decreasing convex function defined for $-\infty<r<+\infty$ satisfying the condition

(A) $\lim _{r \rightarrow+\infty} \varphi(r) / r=+\infty$

and assume that

(B) the subharmonic function $\varphi(v)$ has a harmonic majorant in $R$, where we set $\varphi(-\infty)=\lim _{r \rightarrow-\infty} \varphi(r)$.

Then

(C) the least harmonic majorant $v^{\wedge}$ of $v$ exists and is in $H P^{\prime}(R)$,

(D) the singular measure $d \mu_{S}$ on $\Delta_{1}$ corresponding to the singular part $\left(v^{\wedge}\right)_{S}$ of $v^{\wedge}$ is non-positive,

2) E.D. Solomentsev proved partly the same results as Gårding and Hörmander's in his paper: Izv. Akad. Nauk SSSR (1938), pp. 571-582. 
(E) the least harmonic majorant $(\varphi(v))^{\wedge}$ of the subharmonic function $\varphi(v)$ exists and is quasi-bounded,

and

$$
(\varphi(v))^{\wedge}(z)=\int_{\Delta_{1}} K(z, \zeta) \varphi\left(v^{*}(\zeta)\right) d \omega(\zeta)
$$

Proof. There exists a finite number $c>0$ such that $\varphi(r)$ is strictly increasing for $r>c-1$. Set $v_{\mathrm{c}}=\max (v, c)$. Then $v_{\mathrm{c}}$ and consequently $\varphi\left(v_{\mathrm{c}}\right)$ are subharmonic. Let $\Gamma_{n, c}$ be the set of points $z$ on $\Gamma_{n}=\partial R_{n}$ such that $v(z) \geq c$ holds $(n=1,2, \ldots)$. Then we have

$$
\begin{aligned}
\varphi\left(v_{c}\left(z_{0}\right)\right) & \leq \int_{\Gamma_{n}} \varphi\left(v_{c}(z)\right) d \omega_{n, z_{0}}(z) \\
& =\int_{\Gamma_{n, \mathrm{c}}} \varphi(v) d \omega_{n, z_{0}}+\varphi(c) \omega_{n, z_{0}}\left(\Gamma_{n}-\Gamma_{n, c}\right) \\
& \leq \int_{\Gamma_{n}} \varphi(v) d \omega_{n, z_{0}}+\varphi(c) \\
& \leq h\left(z_{0}\right)+\varphi(c)
\end{aligned}
$$

for arbitrary point $z_{0}$ in $R$, where $h$ is a harmonic majorant of $\varphi(v)$ in $R$. Hence $\varphi\left(v_{c}\right) \leq h+\varphi(c)$ in $R$ and we have $v_{\mathrm{c}} \leq \varphi^{-1}(h+\varphi(c))$, the right hand side being superharmonic, so that $\left(v_{c}\right)^{\wedge} \leq \varphi^{-1}(h+\varphi(c))$, or $\varphi\left(\left(v_{c}\right)^{\wedge}\right) \leq h+\varphi(c)$. The assertion (C) is immediate since $v \leq v_{c} \leq\left(v_{c}\right)^{\wedge}$.

Let $\Phi(r)$ be the restriction of $\varphi(r)$ to $r \geq 0$ and set $u=\left(v_{c}\right)^{\wedge}$. Then from above

$$
\Phi(u)=\varphi\left(\left(v_{c}\right)^{\wedge}\right) \leq h+\varphi(c) .
$$

By de la Vallée Poussin-Doob's lemma, $u$ is U.A.I. for $z_{0}$ and $\left\{R_{n}\right\}$ so that $u$ is a non-negative quasi-bounded harmonic function in $R$. This shows the assertion (D) for $v^{\wedge} \leq u$ implies $\left(v^{\wedge}\right)_{S} \leq u_{S}=0$.

Set $u_{n}=u \wedge n$ for positive integer $n \geq c$ so that $u_{n} \nearrow u$ by the definition. Then we have

$$
\lim _{n \rightarrow+\infty}\left(\varphi\left(u_{n}\right)\right)^{\wedge}=(\varphi(u))^{\wedge} .
$$

In fact, on the one hand, $\left(\varphi\left(u_{n}\right)\right)^{\wedge} \leq(\varphi(u))^{\wedge}$ and on the other hand, $\lim _{n \rightarrow+\infty}\left(\varphi\left(u_{n}\right)\right)^{\wedge} \geq \varphi(u)$, this can be shown as follows. From $\varphi\left(u_{n}\right) \leq\left(\varphi\left(u_{n}\right)\right)^{\wedge}$ 
we have $u_{n} \leq \varphi^{-1}\left(\left(\varphi\left(u_{n}\right)\right)^{\wedge}\right)$ for $u_{n} \geq c$. Consequently $u_{n} \leq \varphi^{-1}\left(\lim _{n \rightarrow+\infty}\right.$ $\left.\left(\varphi\left(u_{n}\right)\right)^{\wedge}\right)$ and so $u \leq \varphi^{-1}\left(\lim _{n \rightarrow+\infty}\left(\varphi\left(u_{n}\right)\right)^{\wedge}\right)$ or $\varphi(u) \leq \lim _{n \rightarrow+\infty}\left(\varphi\left(u_{n}\right)\right)^{\wedge}$.

Now $\left(^{*}\right)$ means that $(\varphi(u))^{\wedge}$ is quasi-bounded. Therefore $0 \leq\left((\varphi(v))^{\wedge}\right)_{S}$ $\leq\left((\varphi(u))^{\wedge}\right)_{S}=0$ which proves our assertion (E).

The last assertion (F) follows from (E) and the continuity of the function $\varphi(r)$.

Using Lemma 3, we can prove our Theorem 2 which is an extension of F. and M. Riesz's theorem ([14], $R$ is the unit open disc and $p=1$ ).

Proof of Theorem 2. "if"-part is obvious. Let $f$ be in the Hardy class $H_{p}(R)$ and set $v=p(\log |f|), \varphi(r)=e^{r}$. Apply Lemma 3 to $v$ and $\varphi(r)$. Obviously the conditions (A) and (B) are satisfied because $\varphi(v)=|f|^{p}$. The conclusion (E) proves our Theorem 2.

5. Let $E$ be a closed polar set in a Riemann surface $R$. It is known that for any bounded and harmonic function $u$ defined in $R-E$ there exists a bounded and harmonic function $\tilde{u}$ defined in $R$ such that the restriction of $\tilde{u}$ to $R-E$ coincides with $u$ ([1], [2]). For clarity, we shall show the following

Lemma 4. Let $E$ be a closed polar set in a Riemann surface $R$ and assume that $u$ is a quasi-bounded harmonic function defined in $R-E$. Then there exists a quasi-bounded harmonic function $\tilde{u}$ defined in $R$ such that the restriction of $\tilde{u}$ to $R-E$ coincides with $u$.

Proof. We can consider only the case $u \geq 0$ (Jordan decomposition in the lattice $\left.H P^{\prime}(R)\right)$. By the definition, $u$ is the limiting function of a monotone non-decreasing sequence of bounded and harmonic functions and vice versa and hence our assertion is immediate.

Proof of Theorem 3. Let $u$ be a quasi-bounded harmonic majorant of $\Psi(|f|)$ in $R-E$. By Lemma 4, $u$ can be continued to $R$ so that the resulting function $\tilde{u}$ is quasi-bounded harmonic in $R$. Consequently $\tilde{u}$ is bounded in any relatively compact open set $G$ in $R$ and hence $f$ is bounded and analytic in $G-E$ because of the property of the function $\Psi(r)$. Hence $f$ can be continued analytically to $R$ and we have the assertions. 
REMARK. We can take as $\Psi(r)$, for example, $r^{p}$ (for $p>0$ ), $\log ^{+} r$, $\log r, \log \left(\log ^{+} r\right),\left(\log ^{+} \log ^{+} r\right)^{p}($ for $p>0), \ldots$, etc.

Corollary 1. (An extension of Tumarkin-Havinson's theorem [17]) Let $E$ be a closed polar set lying in a Riemann surface $R$. If a function $f$ is in the Smirnov class $S(R-E)$, then there exists an analytic function $\tilde{f}$ in the Smirnov class $S(R)$ such that the restriction of $\tilde{f}$ to $R-E$ coincides with $f$.

Proof. This is a consequence of Theorem 1 and Theorem 3 with $\Psi(r)=\log ^{+} r$.

Corollary 2. (Parreau [13], Theorem 20) Let E be a closed polar set lying in a Riemann surface $R$. If a function $f$ is in the class $H_{p}(R-E)$ for $p>0$, then there exists $\tilde{f}$ in the class $H_{p}(R)$ such that the restriction of $\tilde{f}$ to $R-E$ coincides with $f$.

Proof. This is a consequence of Theorem 2 and Theorem 3 with $\Psi(r)=r^{p}$.

Remark. Parreau's theorem can be proved, using Corollary 1 above, if we assume the fact that the polar set $E$ is removable for non-negative superharmonic functions ([1], [2]).

W. Rudin ([15], at p. 49) pointed out that the analogous assertion for the class $A L$ is false.

6. As usual we shall denote by $O_{X}$ the totality of open Riemann surfaces $R$ (including parabolic types) on which the given family $X(R)$ of functions consists only of constants. Then we have

$$
O_{A L} \subset O_{S} \subset O_{H_{p}} \subset O_{A B} \quad(\text { for } p>0) \text {. }
$$

Parreau ([13], p. 192) proved that the inclusion relation $O_{A L} \subset O_{H_{p}}($ for $p>0$ ) is proper, using P.J. Myrberg's example in [10]. Using the fact that one point is removable for the Smirnov class $S$ and the inequality: $\log ^{+}|\alpha-\beta|^{2} \leq$ $2\left(\log ^{+}|\alpha|+\log ^{+}|\beta|+\log 2\right)$, for complex numbers $\alpha$ and $\beta$, we can prove that the inclusion relation $O_{A L} \subset O_{S}$ is proper by the same method as in [10].

\section{REFERENCES}

[ 1 ] M. Brelot: Sur la théorie autonome des fonctions sousharmoniques; Bull. Sc. Math., 65 (1941), 72-98. 
[2 ] M. Brelot: Éléments de la théorie classique du potentiel; Les Cours de Sorbonne, C.D.U., Paris, (1959), 3rd edition (1965).

[3] C. Constantinescu and A. Cornea: Ideale Ränder Riemannscher Flächen; Springer, Berlin, (1963).

[4] J.L. Doob: Probability method applied to the first boundary value problem; Proc. 3rd Berkeley Symp. Math. Statis. Prob., 2 (1956), 49-80.

[5] J.L. Doob: A non-probabilistic proof of the relative Fatou theorem; Ann. Inst. Fourier, 9 (1959), 293-300.

[6] J.L. Doob: Boundary properties of functions with finite Dirichlet integrals; Ann. Inst. Fourier, 12 (1962), 573-621.

[ 7] L. Gårding and L. Hörmander: Strongly subharmonic functions; Math. Scand., 15 (1964), 93-96.

[8] F.W. Gehring: The asymptotic values for analytic functions with bounded characteristic; Quart. J. Math. Oxford, 9 (1958), 282-9.

[ 9 ] M. Heins: Lindelöfian maps; Ann. Math., 62 (1955), 418-446.

[10] P.J. Myrberg: Über die analytische Fortsetzung von beschränkten Funktionen; Ann. Acad. Sci. Fenn. A.I, 58 (1949).

[11] L. Naïm: Sur le rôle de la frontière de R.S. Martin dans la théorie du potentiel; Ann. Inst. Fourier, 7 (1957), 183-281.

[12] R. Nevanlinna: Eindeutige analytische Funktionen; Springer, Berlin, (1953).

[13] M. Parreau: Sur les moyennes des fonctions harmoniques et analytiques et la classification des surfaces de Riemann; Ann. Inst. Fourier, 3 (1952), 103-197.

[14] F. Riesz and M. Riesz: Über die Randwerte einer analytischen Funktion; C.R. Congr. Math. Scand. Stockholm, (1916), 27-44.

[15] W. Rudin: Analytic functions of class $H_{p}$; Trans. Amer. Math. Soc., 78 (1955), 46-66.

[16] V.I. Smirnov: Sur les formules de Cauchy et de Green et quelques problèmes qui s'y rattachent; Izv. AN SSSR, ser. fiz.-mat., 3 (1932), 337-372.

[17] G. Ts. Tumarkin and S. Ya. Havinson: On removal of singularities of analytic functions of a class (class D); Uspehi Matem. Nauk, 12 (1957), 193-9. (Russian).

[18] C. de la Vallée Poussin: Sur l'integrale de Lebesgue; Trans. Amer. Math. Soc., 16 (1915), 435-501.

Mathematical Institute

Nagoya University 